\title{
Réduire les déchets alimentaires par la créativité et la convivialité
}

Les Disco Soupes comme dispositif de transformation des pratiques de conservation

\section{Olivier Wathelet et Jérémy Joncheray}

\section{(2) OpenEdition}

Journals

Édition électronique

URL : https://journals.openedition.org/tc/8826

DOI : $10.4000 /$ tc. 8826

ISSN : 1952-420X

Éditeur

Éditions de l'EHESS

Édition imprimée

Date de publication : 17 mai 2018

Pagination : 220-233

ISBN : 978-2-7132-2750-9

ISSN : 0248-6016

Référence électronique

Olivier Wathelet et Jérémy Joncheray, «Réduire les déchets alimentaires par la créativité et la

convivialité », Techniques \& Culture [En ligne], 69 | 2018, mis en ligne le 17 mai 2020, consulté le 29

septembre 2022. URL : http://journals.openedition.org/tc/8826 ; DOI : https://doi.org/10.4000/tc.8826 


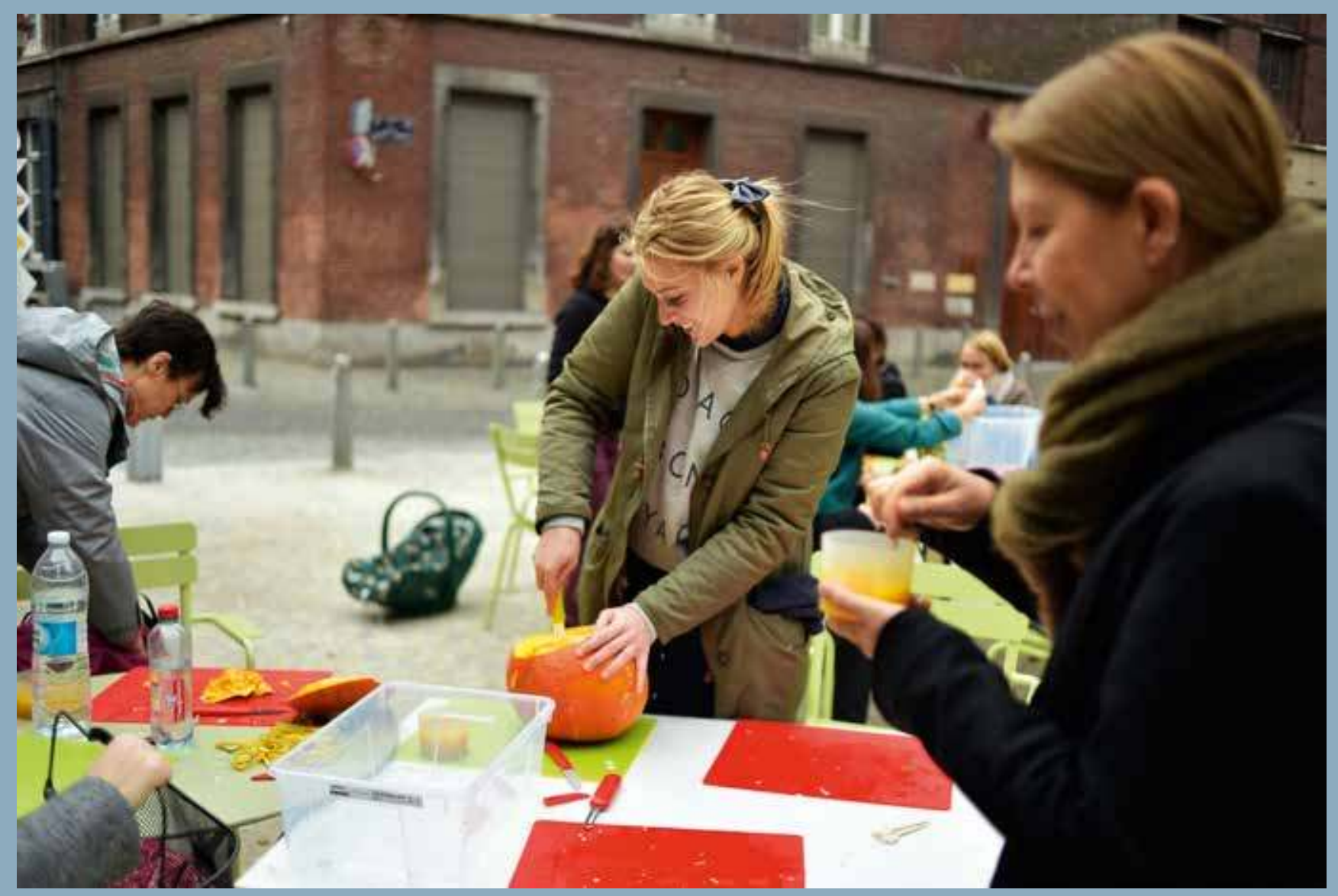




\section{Réduire les déchets alimentaires par la créativité et la convivialité}

\section{Les Disco Soupes comme dispositif de transformation des pratiques de conservation}

Lorsqu'elle n'est pas achetée ou consommée, la nourriture risque de quitter le régime de la conservation pour devenir un déchet, empruntant une trajectoire de requalification marquée par l'ordre normatif qui distingue ce qui est «bon à manger» de ce qui ne l'est pas (Evans 2012, Evans et al. 2012). Les «fruits et légumes moches ${ }^{1} »$ qu'ont promus récemment plusieurs acteurs de la grande distribution en Europe témoignent de l'importance de cette sortie possible du mangeable, tout en révélant l'articulation entre les logiques d'approvisionnements individuels et celles portées par les acteurs industriels (Lhoste \& Stuart 2012) en vue de réduire le «scandale symbolique» que représente le fait de jeter des aliments (Aschemann-Witzel et al. 2015, Caplan 2017).

Considérés à l'échelle de la biographie culturelle des choses (Kopytoff 1986), les déchets alimentaires occupent une position instable entre objets jetables, dons aux «nécessiteux», ou encore matériaux de compostage. À l'instar des rebuts récupérés et revendus par Emmaüs (Hoarau 1999), ils changent progressivement de statut en même temps qu'ils feront l'objet d'un traitement par une série d'acteurs. Et en retour, ils transforment ces derniers en leur faisant expérimenter une nouvelle situation culinaire. Les Disco Soupes sont une association agissant au moment de ce double changement d'état, avec pour vocation de convertir des rebuts en phase de sortie du circuit culinaire en matériaux de nouveau bons à consommer. Dans le cas de l'expérience liégeoise que nous décrivons ici, la précarité alimentaire, que d'autres formes de «soupes populaires» entendent prioritairement prendre en charge, ne fait pas l'objet d'une attention particulière. Il s'agit au contraire de transformer le rapport des citoyens à l'alimentation en mettant en place des évènements urbains, festifs, valorisant des invendus pour créer un repas. Aussi, à la différence du procédé mis en place au sein d'Emmaüs, l'attention est portée sur la convivialité comme mode d'action, et non sur la gestion technique du processus de récupération.

Comment un tel dispositif ambitionne de transformer le champ des pratiques de conservation, et son verso la mise au rebut, au-delà de l'évènement lui-même? Les organisateurs mettent 
Les messages concernant le gaspillage et ses enjeux sont proposés en périphérie de l'activité. En contraste sur ce point avec d'autres, plus explicitement tournés vers des enjeux de justice alimentaire (Caplan 2016, Paddeu 2016), notre démarche vise cependant une pareille transformation des pratiques de conservation alimentaire vers des formes alternatives de consommation.

Juillet 2016 l'accent sur la créativité comme valeur intégratrice mais aussi comme un projet à construire (Ingold \& Hallam 2007) et comme levier de transformation. Le choix de la soupe illustre bien ce paradigme créatif, reposant sur l'élaboration d'un dispositif capable de négocier l'incertitude de l'évènement. Ce plat se prête à de nombreuses associations d'ingrédients, et augmente donc les chances de réaliser une préparation appréciée sur la base de denrées contraintes.

Les premières Disco Soupes ont eu lieu à Berlin, alors ouvertement inspirées de la « Schnippel Disko » organisée par Slow Food Allemagne, avant d'être importées en France et que soit fondée en 2012 une association éponyme. C'est depuis cette attache française que le principe sera ensuite exporté dans d'autres pays d'Europe avec une forte autonomie des acteurs. Le respect d'une liste minimale de principes, les Discommandements ${ }^{2}$, suffit, offrant dès lors un cadre d'engagement idéologique très ouvert. L'initiative vise à créer des occasions de partage alimentaire, urbain et accessible aux passants, de manière à valoriser des rebuts comestibles et démontrer la possibilité de repousser les limites habituelles du cuisinable. Comme en témoigne son slogan,

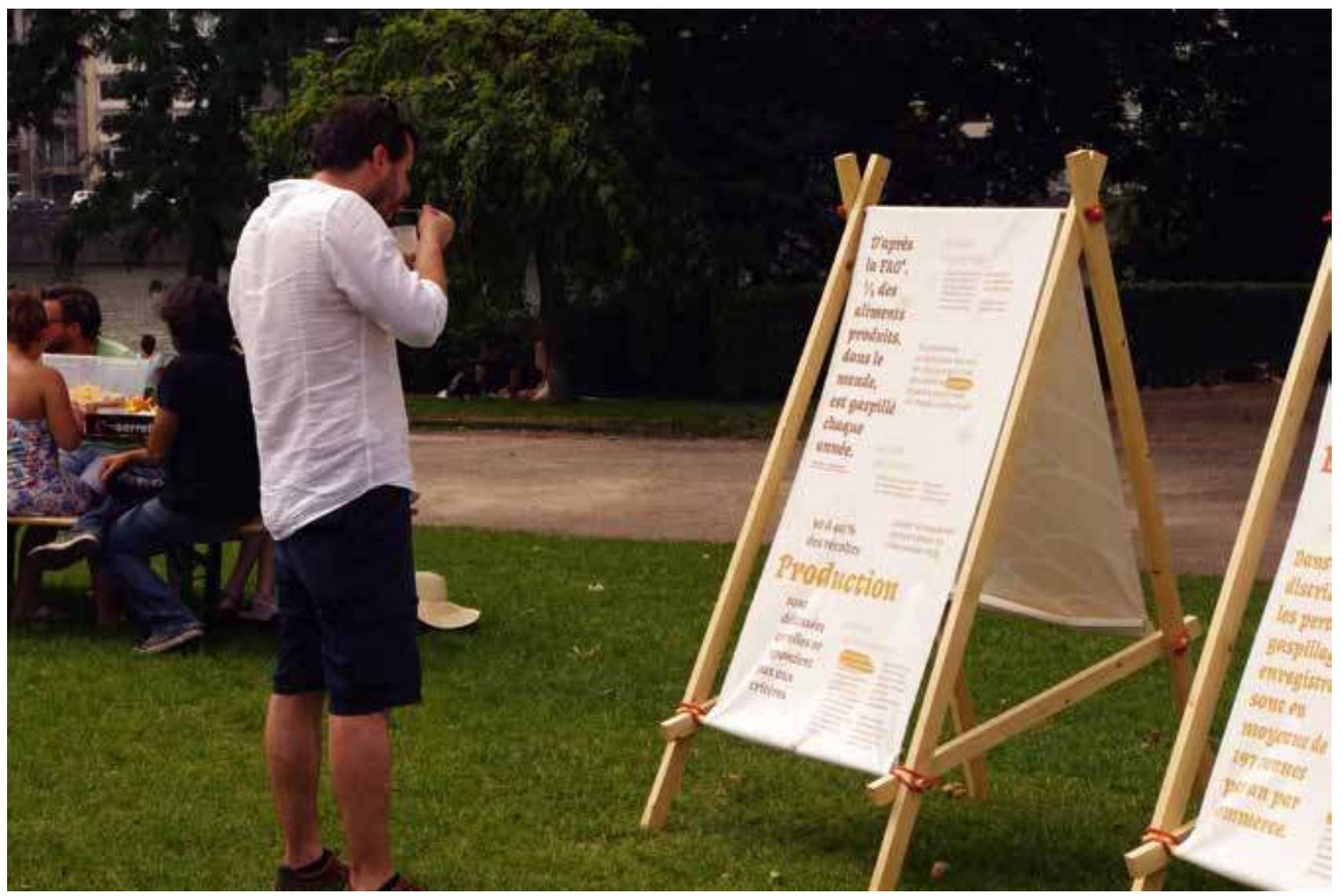




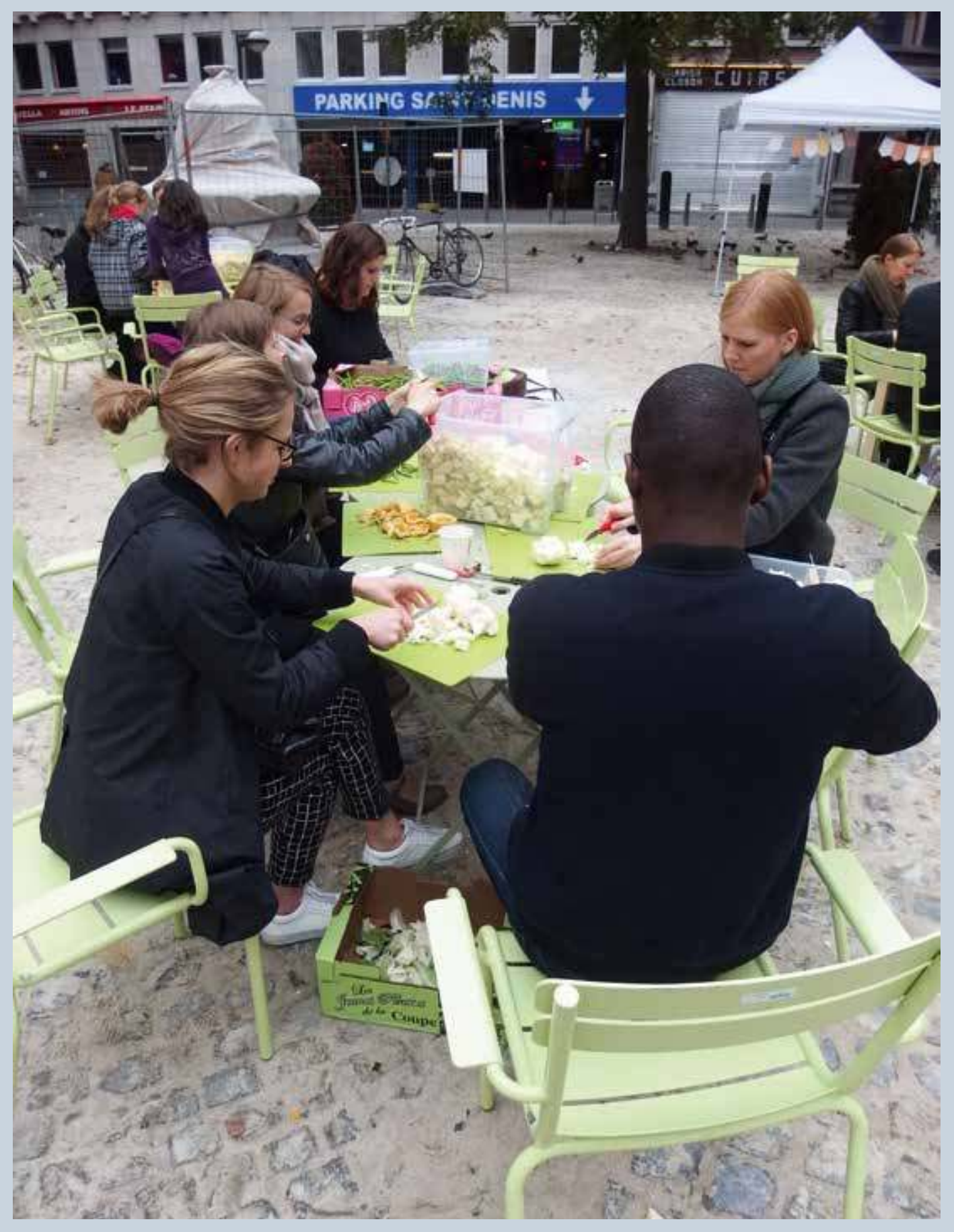

2. Les participants sont invités à travailler de manière méthodique, lente, afin de gérer les temps de cuisson des différents ingrédients et assurer une expérience continue. Octobre 2015 
« la convivialité contre le gâchis », elle privilégie une démarche «positive et non moralisatrice», que la collaboration temporaire entre cuisiniers improvisés permet de générer.

Le schéma général de l'activité repose sur quelques étapes simples. Après avoir fait le tour des grandes surfaces, des distributeurs et maraîchers pour récupérer des rebuts alimentaires (trop petits, trois gros, trop mûrs...), les organisateurs - à Liège, une équipe resserrée autour de deux personnes et d'une dizaine d'amis et relations proches - s'installent, avec une cuisine mobile, dans un lieu préalablement validé par les autorités municipales. Alors, passants et habitués informés par les réseaux sociaux se joignent à eux pour éplucher, découper, détailler, laver ces fruits et légumes afin d'en faire diverses préparations. En fonction des combinaisons rendues possibles par les ingrédients disponibles seront cuisinées des soupes, mais également des smoothies, des salades, purées, compotes. Tout est ensuite distribué gratuitement, en compagnie de la présence sonore de musiciens destinés à faire signal au sein de l'espace public, et à rendre festif l'évènement.

Les denrées collectées sont sélectionnées de manière à privilégier les aliments de saison, celles qui tendent à se périmer le plus vite (logique éthique) et celles qui autorisent des associations de goûts culturellement cohérentes, permettent une cuisson «minute» et demandent un équipement rudimentaire $^{3}$ (logique pratique), la cuisine devant se faire dans la rue. Le travail de sélection est donc une première étape d'extension de la conservation reposant sur des savoirs accumulés.

3. Une Disco Soupe en bord de Meuse, lors de l'inauguration du nouveau musée La Boverie, à Liège. Mai 2016

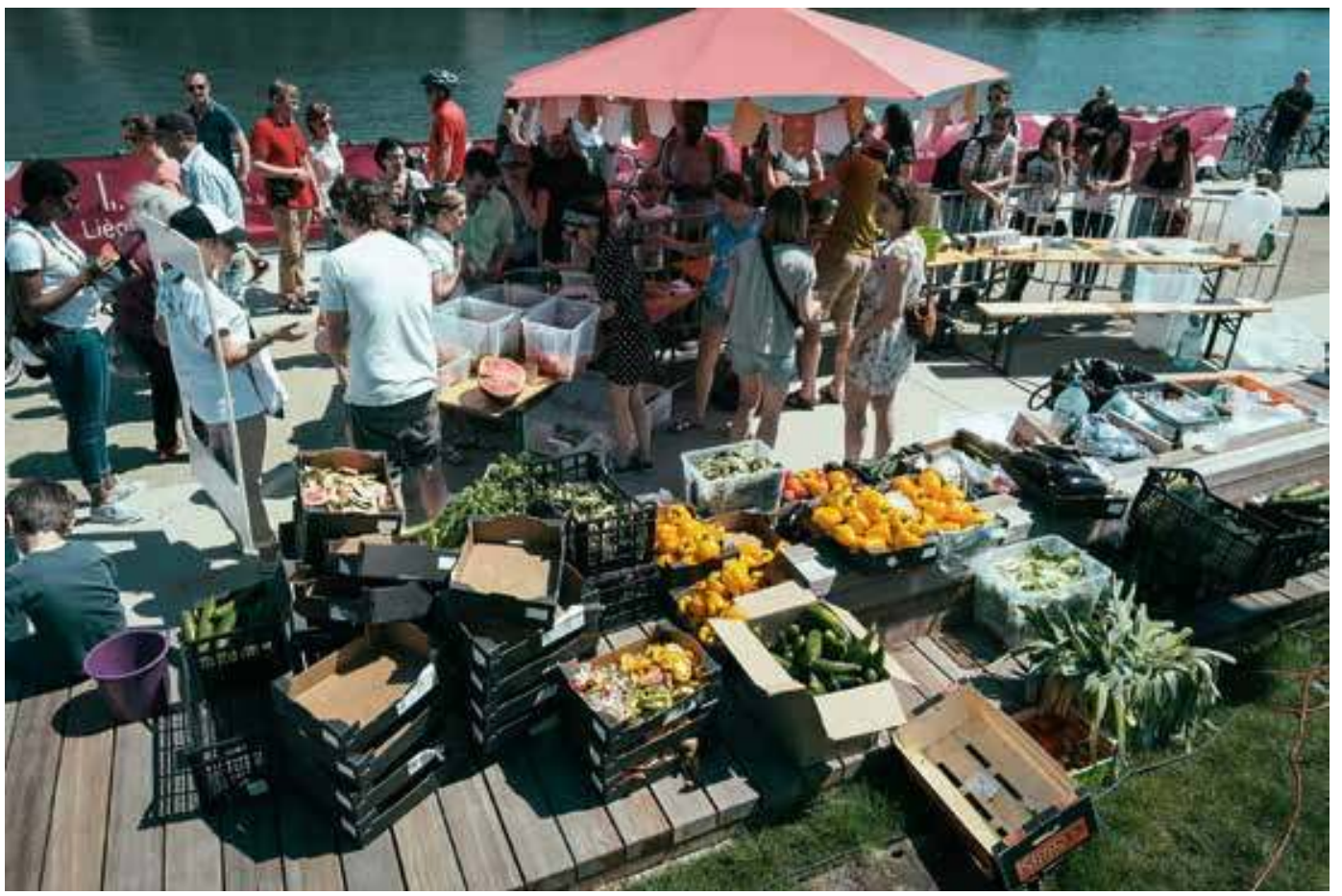




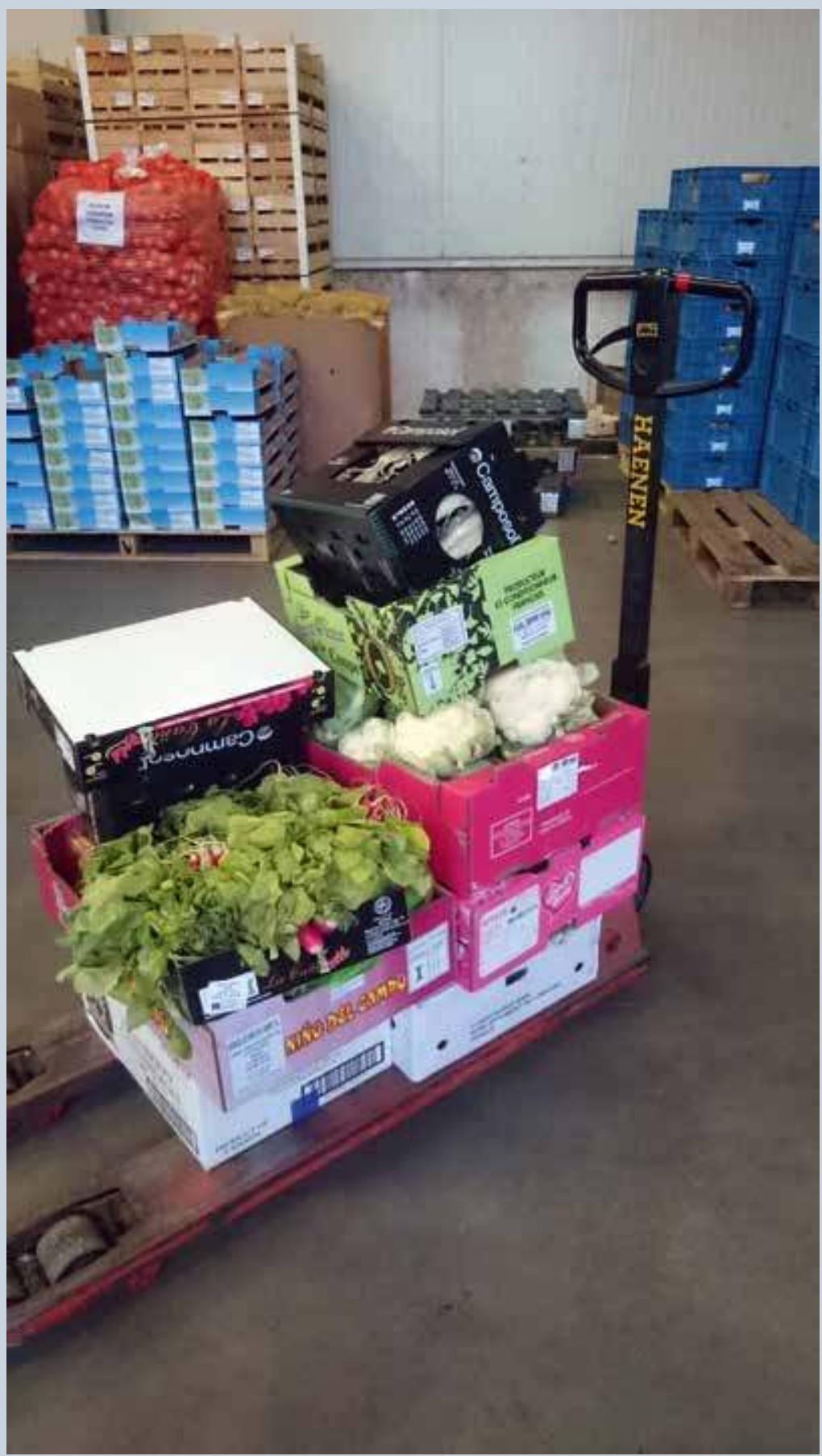

4. Un échantillon d'une séance de récupération de légumes auprès d'un grossiste, réalisée avec la collaboration souvent enthousiaste des em. ployés des dépôts visités. Novembre 2017. 


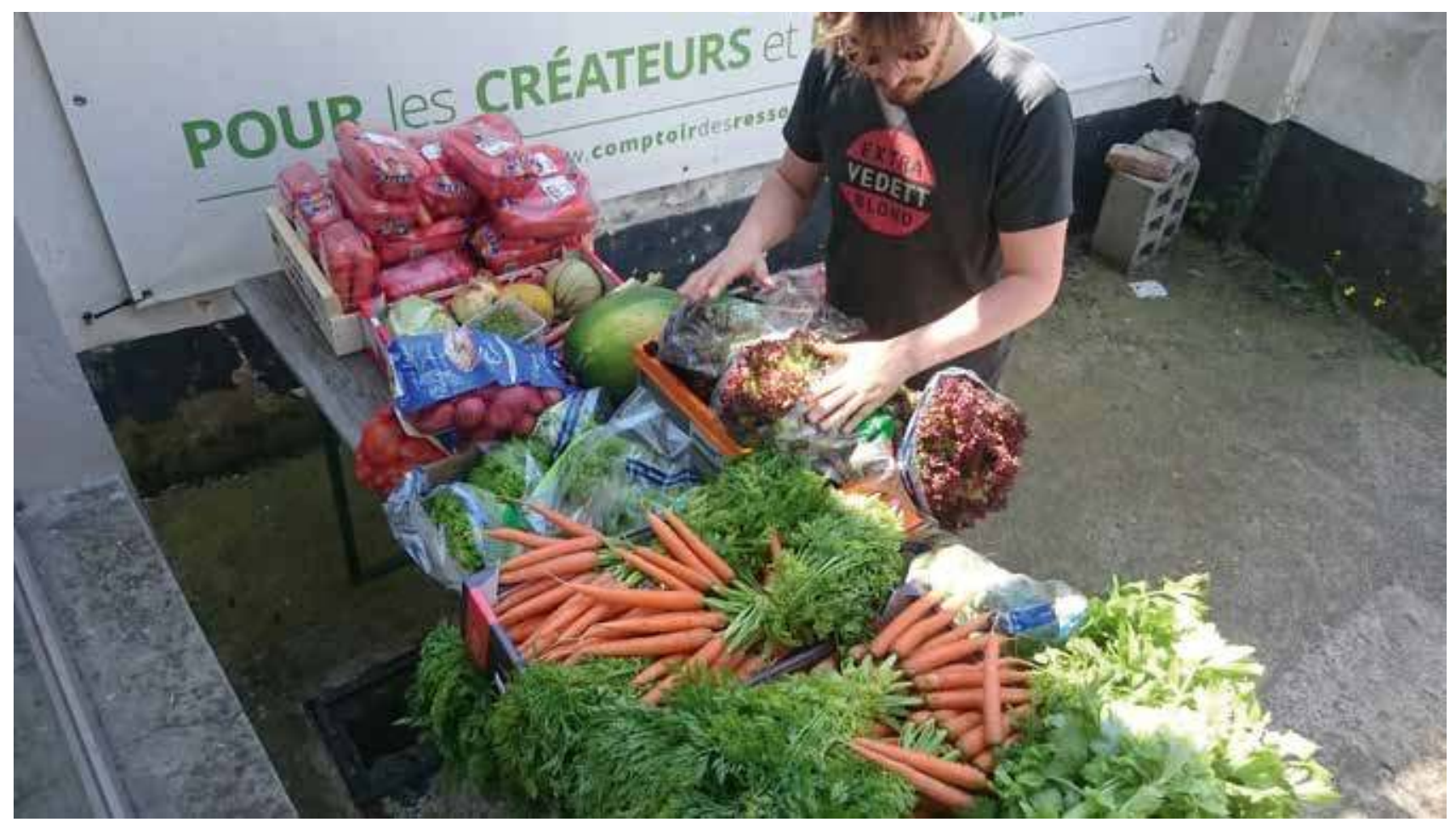

5. Les fruits et légumes sélectionnés sont stockés ensuite, de quelques minutes - lorsqu'il s'agit de récupérer ce qui est disponible en fin de marché-, à deux ou trois jours dans une cave ou un lieu mis à disposition par un membre de l'association. S'engage alors un long et méticuleux travail de tri. II va du nettoyage au conditionnement pour l'évènement du lendemain.

20 juillet 2016 , veille d'une Disco Soupe.
De même, les rebuts pris en charge subissent une première étape de transformation de manière à leur «donner une bonne tête», pour qu'ils soient choisis par les participants des Disco Soupes et qu'ils ne se gâchent pas par propagation durant le stockage intermédiaire. Le principe technique dominant est de créer les conditions de la consommation de différentes façons, de manière à réduire au maximum le risque de perte. Ce principe vaut tout au long de l'évènement. Ainsi, les rebuts qui ne seront pas consommés lors de la Disco Soupe seront remis en circulation de manière à préserver leur nouvelle qualité de consommable le plus longtemps possible, selon différents scénarios. L'intervention culinaire apparaît comme un mécanisme d'extension de la «date limite de consommation» initialement fixée.

Durant l'acte culinaire lui-même, c'est l'économie du geste qui est pensée pour répondre à cet impératif de réduction du déchet. Il s'agit de valoriser au mieux chaque produit récolté par des découpes différentes et un usage de toutes les parties du légume. Des fanes de carotte on fera de la soupe, des morceaux allongés des apéritifs crus et de morceaux plus petits, une compotée. Les solutions créatives pour augmenter la conservation des aliments sont mises en œuvre aux différentes étapes des évènements et en constituent une démonstration en acte. Elles ne reposent pas sur des procédés de stockages plus techniques et plus efficaces, mais sur une augmentation des compétences de valorisation culinaire. 
6. Lorsqu'il reste simplement les épluchures et autres déchets peu valorisables, un partenaire (producteurs, particuliers, éleveurs de cochons) les récupère afin de les composter. Lorsqu'il s'agit de petites quantités d'aliments, les organisateurs proposent aux participants d'emporter des petites quantités chez eux. Si de grosses quantités sont disponibles, elles seront offertes à des œuvres caritatives. Enfin, les plats préparés qui ne sont pas consommés sont redistribués dans l'équipe ou remis directement dans le réfrigérateur du lieu où le matériel est stocké, qui est un espace de travail partagé. Des packs de conditionnement de taille restreinte sont proposés en fin de Disco Soupe pour faire circuler les restes tout en évitant une accumulation disproportionnée au regard des capacités culinaires, susceptible de favoriser un nouveau déchet. Octobre 2015.

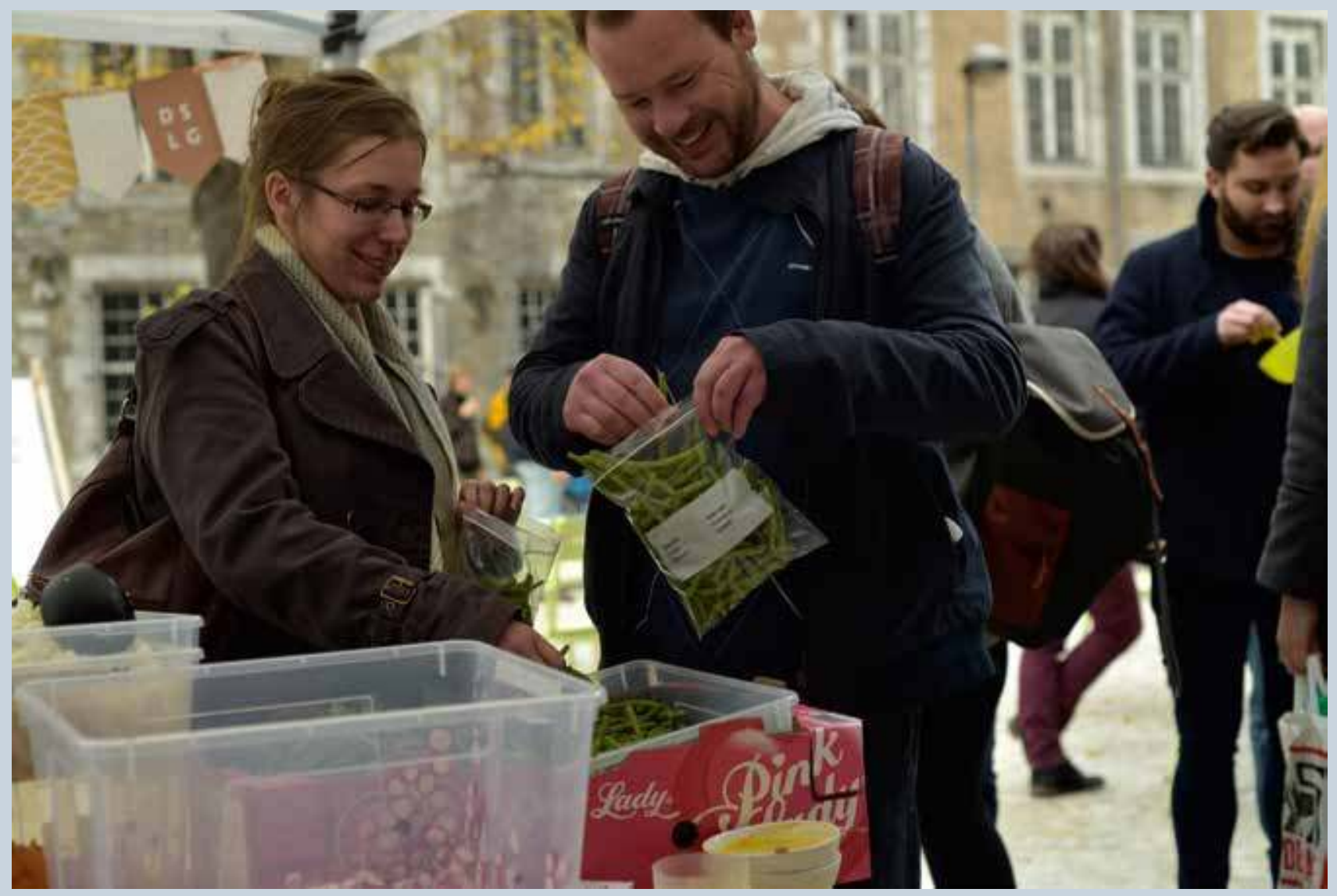




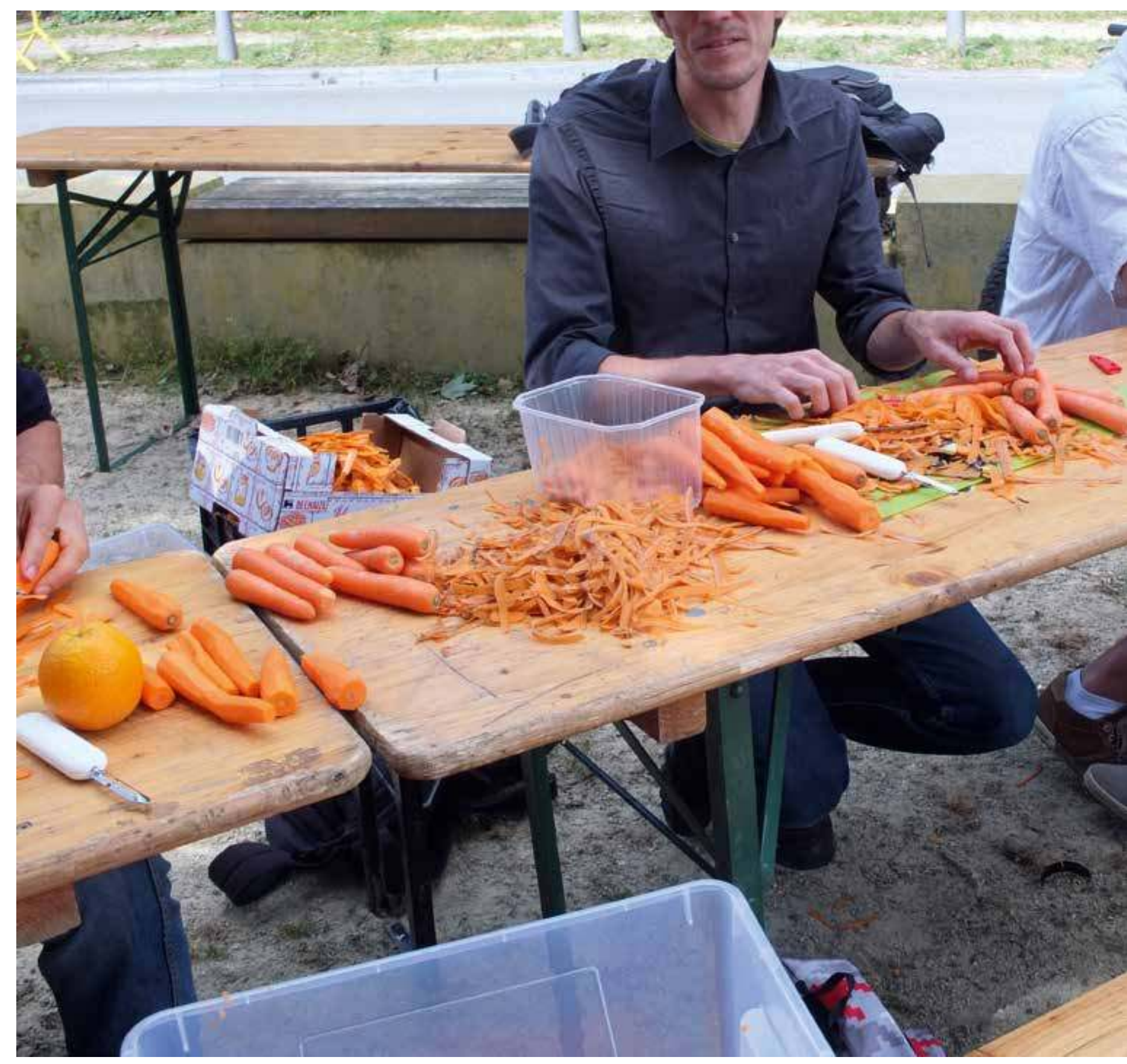




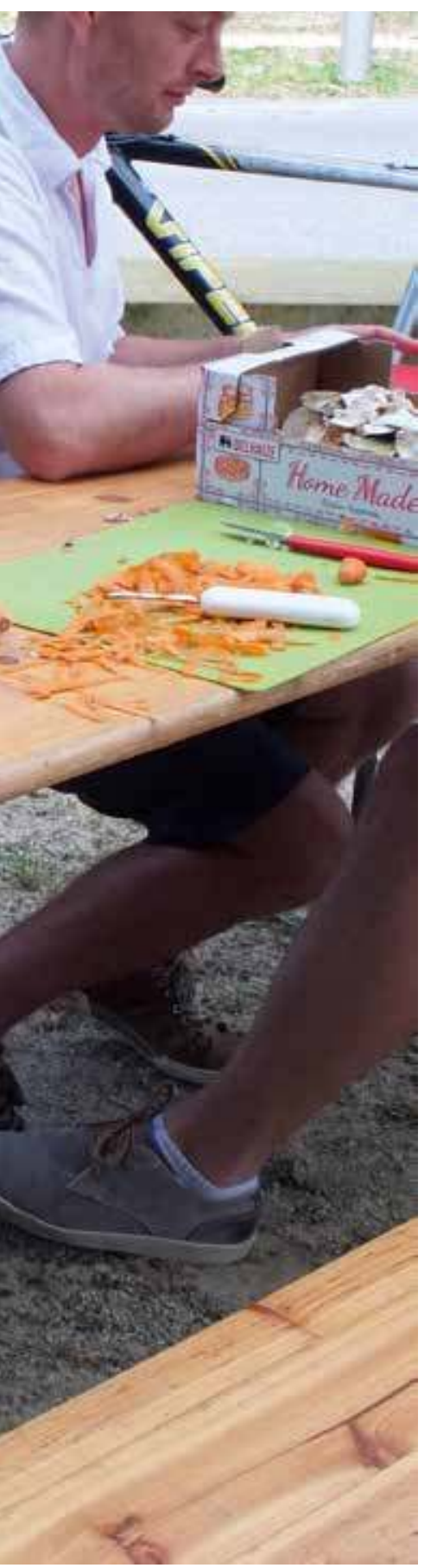

Le dispositif combine donc des stratégies pour maximaliser la valorisation des rebuts, d'une part, et pour rendre attrayant et pratique l'engagement des participants, d'autre part. La mise en œuvre de ce double levier est cependant fragile. Il est arrivé que l'évènement ne fonctionne pas, en raison d'une météo maussade ou d'un mauvais emplacement, trop peu passant. À une autre occasion, une Disco Soupe a été annulée la veille, car il y avait eu un souci de communication avec les producteurs et les grossistes devant apporter leurs rebuts, l'ensemble des marchandises ayant été jetées "par erreur» quelques heures avant le passage des organisateurs pour les récupérer.

Les Disco Soupes apparaissent dès lors comme des dispositifs visant à suggérer de nouvelles pratiques de conservation alimentaire en mobilisant la convivialité et la créativité comme double levier pour engager les publics. En générant un moment festif, et en traitant les rebuts pour les rendre le plus aisément transformables, les organisateurs ambitionnent une performance efficace et reproductible. La convivialité du cadre est au service d'une requalification du bon à manger qui est démontrée, et expérimentée, par la réalisation d'une performance créative partagée et dans une certaine mesure collaborative. Elle offre un cadre pour mettre à l'arrière-plan les enjeux de la «sécurité alimentaire » et privilégier le savoir-faire de préparation.
7. Ce jour-là, les carottes récupérées présentaient un calibre adéquat à la vente, mais leur filet était percé. Elles seront préparées en purée. Août 2016. 


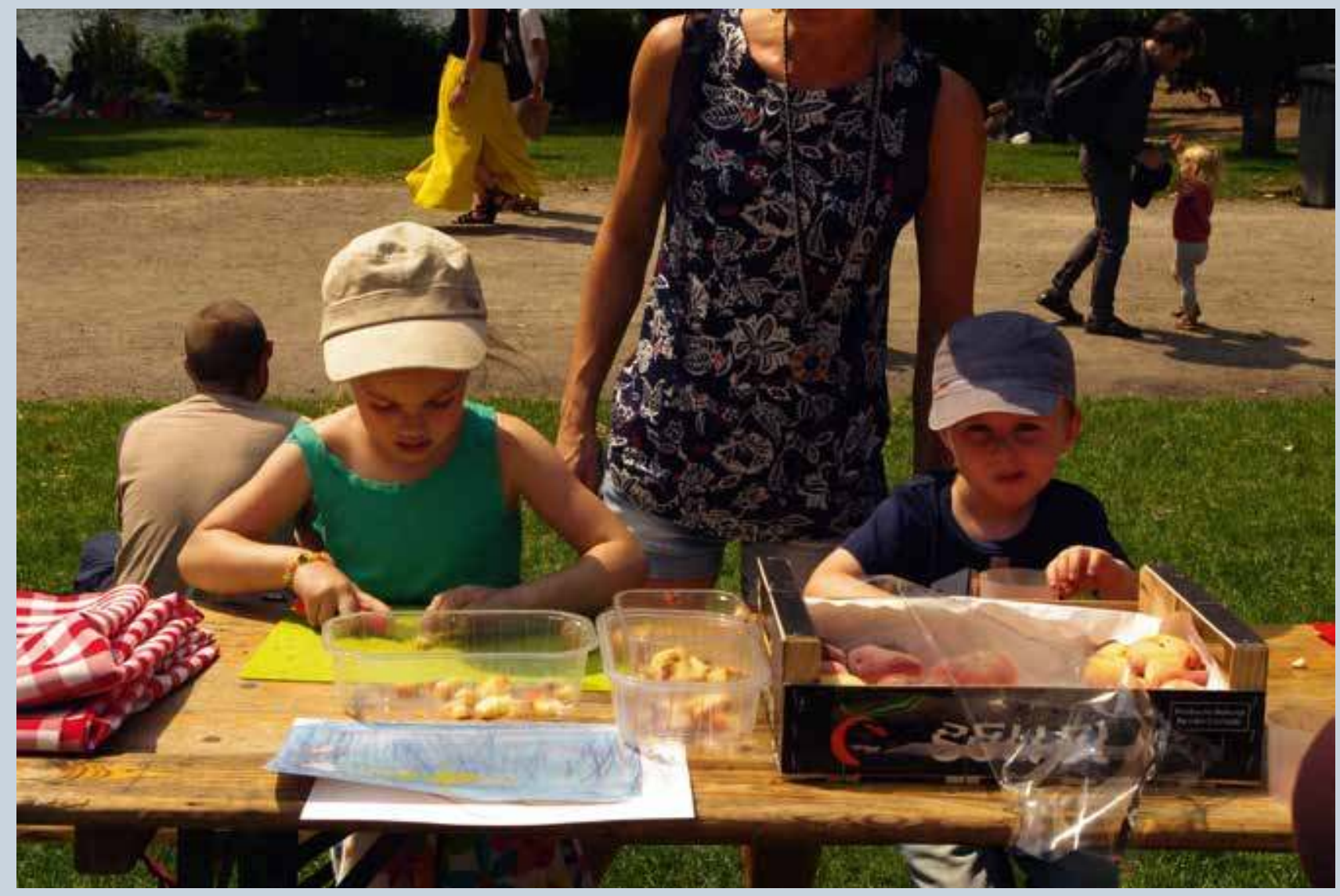

8. L'aspect transgénérationnel est important pour les organisateurs. Ils considèrent la transmission du bien manger et du mieux manger comme un moyen de faire changer les comportements sur ce qui est préparé. Le public réuni varie également très fortement d'un évènement à l'autre. Lorsque ces derniers sont réalisés en lien avec d'autres projets (inauguration, évènement artistique...), les participants sont plus nombreux et plus diversifiés. D'autres fois, l'évènement est plus restreint et se limite à des personnes déjà convaincues par l'action. On touche peut-être ici à une spécificité des «consommations engagées» contemporaines (Dubuisson-Quellier 2009) qui reposent en grande partie sur une qualité d'expérience et non prioritairement sur un parti pris idéologique, rendant très instable la forme et l'intensité des engagements. Juillet 2016 . 
9. II n'est pas rare que les usagers des Disco Soupes sollicitent les recettes réalisées. Improvisées pour partie, elles sont écrites pour l'occasion. Elles permettent la transmission de la performance au-delà de son cadre original. «Finalement, expliquent les organisateurs, notre objectif est de ne plus pouvoir faire de Disco Soupe par manque de matière. Nous aimerions un jour, arriver chez les grossistes et entendre: «Ah, malheureusement on a plus de déchet, tout est valorisé par ceci, cela » ou encore chez un producteur: «Les produits hors calibres sont valorisés par une association qui fait des conserves et de la réinsertion... ». On verra d'ici combien de temps nous y parviendrons.... Août 2016.

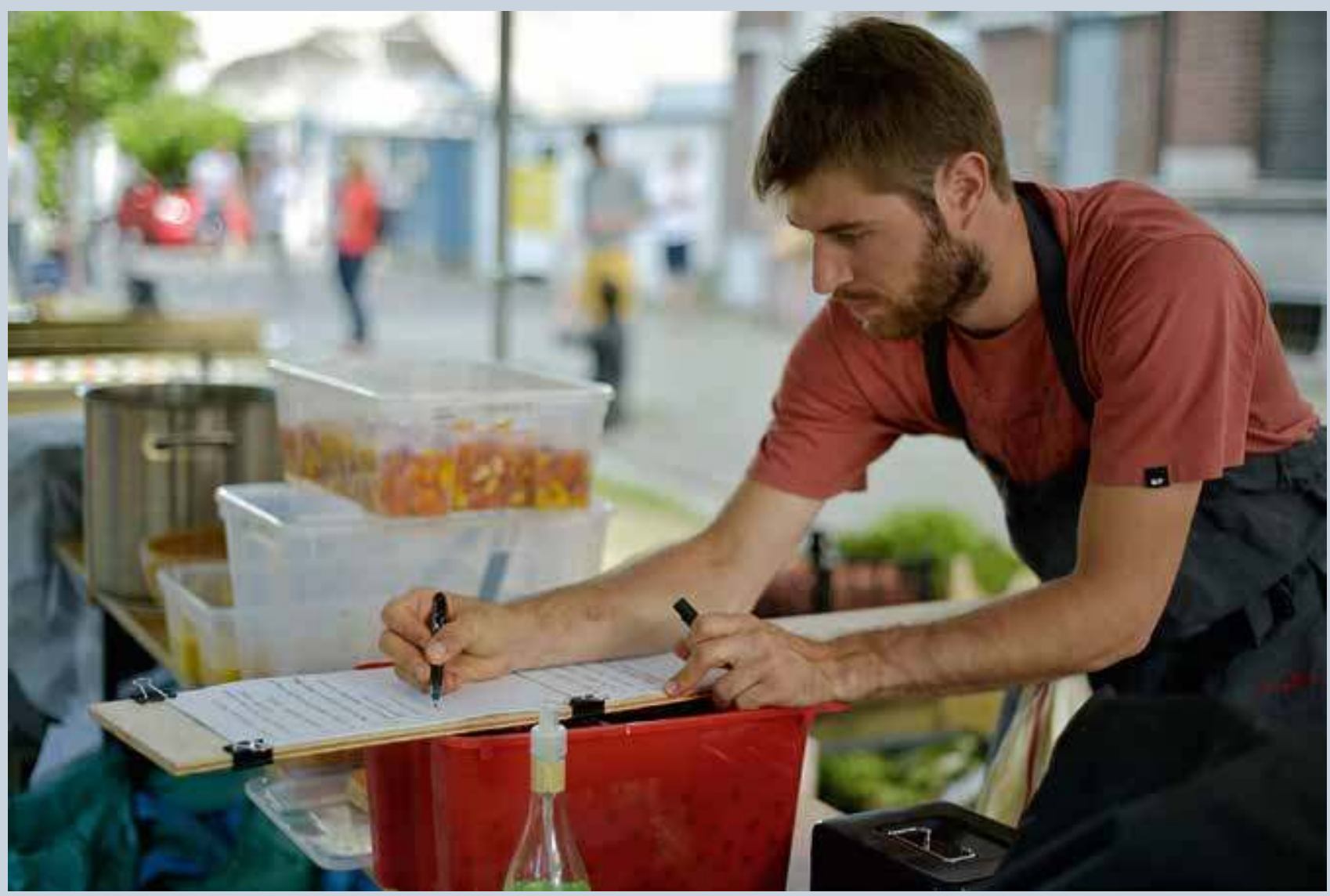


On peut cependant se demander à quel point cette démarche procède, comme l'a montré François Hoarau (1999), d'une transformation du sujet qui, en collectant, mettant en ordre, transformant, agirait sur lui-même également? Autrement dit, que reste-t-il une fois l'évènement achevé, en particulier auprès de ceux qui sont d'ores et déjà engagés au quotidien dans de telles démarches? Et quel effet cela a-t-il en termes de réorganisation durable des pratiques alimentaires, à l'articulation entre les sphères privées, économiques et associatives. Plus largement, cet exemple brièvement esquissé, nous invite à interroger les pratiques actuelles de consommation responsables qui, en marge d'une certaine idée « classique» des pratiques militantes, mobilisent la créativité des acteurs comme ressource pour engager une transformation du monde social.

\section{Notes}

1. https://theconversation.com/les-legumes-moches -une-belle-idee-49616.

2. http://discosoupe.org/la-charte-et-les-valeurs/.

3. L'équipement se résume à deux grandes malles en bois pour les produits comestibles et les accessoires de cuisine d'une part, et le matériel «logistique»

d'autre part (pharmacie, décoration, supports de communication), un bruleur à gaz et une batterie de casseroles. Occasionnellement, un matériel de seconde main est acquis pour rendre possible des préparations plus complexes.

\section{Iconographie}

Image d'ouverture. Entre dégustation et préparation, le public est invité à participer activement à l'évènement. Octobre 2015. @ Antonio Gomez Garcia.

1, 3, 4, $5 \& 8$. C Disco Soupe, Liège.

2, 6, $7 \&$ 9. (C) Antonio Gomez Garcia.

\section{Les auteurs}

Olivier Wathelet est docteur en anthropologie de l'Université de Nice-Sophia Antipolis. Sa thèse a porté sur l'anthropologie cognitive des odeurs en contexte familial, en France. Depuis lors, il s'est intéressé à l'étude des pratiques culinaires professionnelles et domestiques, et aux démarches d'innovation centrées sur les utilisateurs au sein de l'agence Users matter. Jérémy Joncheray est designer graphique au sein de l'atelier qu'il a fondé Signes du quotidien. En parallèle, sa pratique est nourrie par des recherches autour de la visualisation des saveurs, notamment en tant que cofondateur des Disco Soupes Liège.

\section{Références}

Aschemann-Witzel, J., de Hooge, I., Amani, P., BechLarsen, T. \& M. Oostindjer 2015 «Consumerrelated food waste: causes and potential for action», Sustainability $7:$ 6457-77.

Caplan, P. 2016 « Big society or broken society? Food banks in the UK», Anthropology Today 32 (1): 5-9.
- 2017 «Win-win? Fodo poverty, food aid and food surplus in the UK today», Anthropology Today 33 (3): $17-22$.

Dubuisson-Quellier, S. 2009 La consommation engagée. Paris: Presse de Sciences Po.

Evans, D. 2012 Food Waste. Home Consumption, Material Culture and Everyday Life. Londres: Bloomsbury. 
Evans, D., Campbell, H. \& A. Murcott 2012 «À brief pre-history of food waste and the social sciences », The Sociological Review 60 (52) : 5-26.

Hoarau, F. 1999 «La communauté Emmaüs de Besançon. Des sujets et des objets pris dans des réseaux d'actions sur les actions » in M.-P. Julien \& C. Rosselin dir. Le sujet contre les objets... tout contre. Ethnographie de cultures matérielles. Paris: Éditions du CTHS: 265-289.

Ingold, T. \& E. Hallam dir. 2007 Creativity and Cultural Improvisation. Londres: Bloomsbury.
Kopytoff, I. 1986 «The cultural biography of things. Commoditization as process » in A. Appadurai dir. The social life of things. Commodities in cultural perspective. Cambridge: CUP: 64-91.

Lhoste, B. 2012 La grande sur-bouffe. Préface par Tristram Stuart. Paris: Rue de l'échiquier.

Paddeu, F. 2016 «D'un mouvement à l'autre: des luttes communautaires de justice environnementale aux pratiques alternatives de justice alimentaire», Justice spatiale - spatial Justice 9: 1-36.

\section{Pour citer l'article}

Wathelet, O. \& J. Joncheray 2018 «Réduire les déchets alimentaires par la créativité et la convivialité. Les Disco Soupes comme dispositif de transformation des pratiques de conservation», Techniques $\mathcal{C}$ Culture 69 «Le temps des aliments», p. 220-233. 